\section{AB1036 FEATURES OF KIDNEY FUNCTION AND URODYNAMICS AT PATIENTS WITH CHRONIC GOUT BASED ON COMPLEX RENAL SCINTIGRAPHY DATA}

M. Gromova ${ }^{1}$, O. Kislyak ${ }^{1}$, V. Tsurko ${ }^{1}$, A. Kashkadayeva ${ }^{2}$, S. Averinova ${ }^{2}$, S. Shiryaev ${ }^{2} .{ }^{1}$ PIROGOV RUSSIAN NATIONAL RESEARCH MEDICAL UNIVERSITY (RNRMU); ${ }^{2}$ N.N.Blokhin National Medical Research Center, Moscow, Russian Federation

Background: One of the frequent manifestations of gout is the gout nephropathy For assessment of the urinary system functional reserves and the risk of renal failure routine analyses of urine in combination with a sonography are often not enough. Modern technology of the systemic examination of nephrourological status based on complex renal scintigraphy (SENS-CRS) was developed in the laboratory of radioisotope diagnosis in "N.N. Blokhin National Medical Research Centre" and realised as an automated workplace. SENS-CRS technology is designed for assessment of the urinary system functional reserves and the risk of renal failure at all macrostructural levels, and allows lowest radiation doses (0.6 mSv for one patient)

Objectives: To define features of kidney function and urodynamics at patients with chronic gout based on the complex renal scintigraphy (CRS) data.

Methods: 59 medical records of patients with gout (2007-2011) were analysed retrospectively. Most of the patients (95\%) were men, average age was $54.4 \pm 9,5$ years. Duration of the disease was $8^{4-11}$ years. All patients had chronic gouty arthritis, $28 \%$ of patients had tophuses. The CRS tests was carried out on a twodetector gamma camera with simultaneous 2 projections recording. ${ }^{99 \mathrm{~m}} \mathrm{Tc}$-technephore was used, a Russian radiopharmaceutical (RP) from the group of bisphosphonates that has hemodynamics of a glomerulotropic product, concentrating mainly in the nephrons via filtration, with partial $(10 \%-15 \%)$ involvement of secretion. Working protocol consisted of a base $21 \mathrm{~min}$ (1 min angiophase) study with administration of RP and a delayed 21 min study without administration of $\mathrm{RP}$, but after taking $200-300 \mathrm{ml}$ of water and/or an antispasmodic or diuretic drug to identify persistent urodynamic dysfunction. The interpretation of CRS data is based on a concentrational-hydrodynamic model of urinary excretion and SENSCRS software. The Statistica 10.0 software was used too.

Results: According to CRS tests patients with gout had, on average, the level of blood cleansing from RP reduced slightly with a trend to a moderate level, and buffer retention of RP labelled blood in extrarenal structures increased. The signs of a relative hemostasis were found against the background of fast excretion accelerated by taking hypotensive drugs. Quantitative analysis of CRS data allows to estimate sustainability of relative urine delayes in the pyelocaliceal system (PCS): in $70 \%$ of patients residual urostasis in the renal parenchyma and groups of calyx remained relatively stable, and the urostasis signs in the renal pelvis were disappearing. This result means that there could be a latent increased residence time of substances such as uric acid as well as nephrotoxic drugs in the kidney parenchyma. This requires control of correct drugs dosage and when prescribing repeated therapy courses.

Conclusions: The SENS-CRS technology provides the quantitative assessment of kidney blood cleansing from RP and concentrational function of parenchyma as well as unique quantitative indicators of urodynamic delays in all parts of urinary tract. This kind of functional diagnostics allows to monitor parenchima and urinary tract condition promptly and with lowest radiation doses, apply therapeutic measures to prevent more severe kidney dysfunction and refer patients to a specialist consultation.

Disclosure of Interest: None declared

DOI: 10.1136/annrheumdis-2018-eular.1342

\section{AB1037 RELATIONSHIP BETWEEN CALCIUM PYROPHOSPHATE DIHYDRATE CRYSTAL AND OPERATED KNEE OSTEOARTHRITIS -GENDER SPECIFIC ANALYSES-}

M. Nishikawa, K. Takami, H. Owaki, T. Fuji. Orthopaedic Surgery, JAPAN COMMUNITY HEALTHCARE ORGANIZATION OSAKA HOSPITAL, Osaka City, Osaka, Japan

Background: Calcium pyrophosphate dihydrate (CPPD) crystal deposition disease is a common arthritic disorder in mainly elderly patients. CPPD crystals are found in multiple joints in association with severe cartilage degeneration in osteoarthritis (OA). The knee is a key target site for both CPPD crystals and OA. CPPD crystals are also one of the common types of crystals causing crystal-induced inflammatory arthritis. There is a need to elucidate factors that can cause progression of knee OA. To address this question, we investigated the relationship between CPPD crystals and operated knee OA.

Objectives: We investigated the relationship between CPPD crystal and operated knee $\mathrm{OA}$ separated by gender.

Methods: Three hundred sixty-six unicompartmental knee arthroplasty (UKA), total knee arthroplasty (TKA) and high tibial osteotomy (HTO) (average age 73.3: male 59: female 307) were performed for over grade III knee OA classified by Kellgren-Lawrence grading scale from 2010 to 2017. At the operation, joint fluids were collected and elucidated the CPPD crystal using polarising microscope. We evaluated the relationship between CPPD crystals, and age, body mass index (BMI), C-reactive protein (CRP), erythrocyte sedimentation rate (ESR), matrix metalloproteinase-3 (MMP-3) at the operation. We defined the osteophyte formation degree (OFD) as grade $0-3^{1}$. We also defined the lower extremity alignment as varus (femorotibial angle: $\left.\mathrm{FTA} \geq 180^{\circ}\right)$, neutral $\left(170^{\circ} \leq \mathrm{FTA}<180^{\circ}\right)$ and valgus $\left(\right.$ FTA $\left.<170^{\circ}\right)$. The relationship between CPPD crystals, and osteophyte formation and lower extremity were also evaluated.

Results: CPPD crystals were detected from $101 \mathrm{Knee} \mathrm{OA} \mathrm{(27.6 \% ).} \mathrm{CPPD(+)} \mathrm{rate}$ in female $(30.0 \%$ : $92 / 307)$ was significantly higher than that in male $(15.3 \%$ : $9 / 59)$. There was a significant difference between $\operatorname{CPPD}(+)$ and (-) in male about only FTA $\left(186.9^{\circ} / 182.0^{\circ}\right)$. There were significant differences between CPPD $(+)$ and $(-)$ in female about age $(76.3 / 72.4)$, FTA $\left(182.7^{\circ} / 183.2^{\circ}\right)$, BMI (24.9/26.6). The more severe OFD became, the higher $\operatorname{CPPD}(+)$ rate was in female, significantly. CPPD $(+)$ rate in valgus knees $(60.9 \%)$ was higher than that in varus knees $(29.6 \%)$ in female significantly.

Conclusions: The European League Against Rheumatism (EULAR) reported that female gender was not a risk factor associated with CPPD crystals ${ }^{2}$. In contrast, a recent study using a relatively large sample size of Japanese cadaveric knees showed a significant correlation for CPPD crystals and female gender ${ }^{3}$ Our data also showed a significantly high $\operatorname{CPPD}(+)$ rate in females. Because our data were based on Japanese patients only, Japanese ethnicity may influence the gender difference in the $\mathrm{CPPD}(+)$ population. In addition, the significantly different items in male and female were different. These results may suggest that the mechanism of CPPD deposition may be different by gender.

\section{REFERENCES :}

[1] Nagaosa $Y$, et al. Development of a logically devised line drawing atlas for grading of knee osteoarthritis. Ann Rheum Dis. 2000;59:587-95.

[2] Zhang W, et al. European League Against Rheumatism recommendations for calcium pyrophosphate deposition. Part I: terminology and diagnosis Ann Rheum Dis. 2011;70:563-70.

[3] Ryu K, et al. The prevalence of and factors related to calcium pyrophosphate dehydrate crystal deposition in the knee joint. Osteoarthritis Cartilage. 2014;22:975-9.

Disclosure of Interest: None declared

DOI: 10.1136/annrheumdis-2018-eular.1235

\section{AB1038 PHARMACOKINETICS, PHARMACODYNAMICS AND SAFETY OF NC-2500, A NOVEL XANTHINE OXIDOREDUCTASE INHIBITOR, IN HEALTHY JAPANESE MALE SUBJECTS}

M. Hirano ${ }^{1}$, S. Kobayashi ${ }^{2}$, E. Miyayama ${ }^{2}$, T. Ohta ${ }^{1}$, M. Yamamoto $^{2}$,

T. Yamakawa'. . Discovery Research Laboratories, Nippon Chemiphar Co., Ltd., Misato, Saitama Prefecture; ${ }^{2}$ Development Planning Department, Nippon Chemiphar Co., Ltd., Tokyo, Japan

Background: Gout flare due to rapid urate reduction after initiating urate-lowering therapy (ULT) is one of the major issues in the therapy, which impairs patients quality of life and adherence. For the prevention of it, the guidelines in the ACR, the EULAR and Japan recommend initiating ULT with a low starting dose followed by adequate titrations, however it is rare in clinical settings. NC-2500 is a novel orally active xanthine oxidoreductase (XOR) inhibitor in development for the treatment of gout/hyperuricemia. Preclinical studies showed that multiple doses increase the plasma concentration and enhance the urate-lowering effect of NC2500 , suggesting that NC-2500 may decrease the risk of gout flare when initiating the treatment.

Objectives: The aim of this study was to evaluate the pharmacokinetics, pharmacodynamics and safety of NC-2500 in healthy Japanese male subjects.

Methods: A Phase 1, randomised, single-blind, placebo-controlled, single and multiple ascending dose study was conducted. Each cohort consisted of 8 subjects, with 6 receiving NC-2500 and 2 receiving placebo orally. A total of 5 cohorts were studied in the single-dose study (10 mg to $160 \mathrm{mg}$, fasted conditions) and 4 cohorts were studied in the multiple-dose study (10 mg to $80 \mathrm{mg}$, fed conditions) The levels of NC-2500 and urate in plasma/serum and urine were assayed at predetermined time points. Safety and tolerability were assessed by physical examination, vital signs, electrocardiography, clinical laboratory tests and adverse events (AEs).

Results: Following singe oral doses of NC-2500, maximum plasma concentration (Cmax) and area under the plasma concentration-time curve (AUC) increased approximately in a dose-proportional manner except that the increase in the Cmax at $160 \mathrm{mg}$ was less than dose proportional. The time to reach the Cmax (Tmax, median) was at 2.0-3.5 hours post dose and food intake delayed the Tmax by 1 hour. In the presence of food, NC-2500 absorption appeared to decrease slightly or not be affected. Plasma NC-2500 concentration increased 